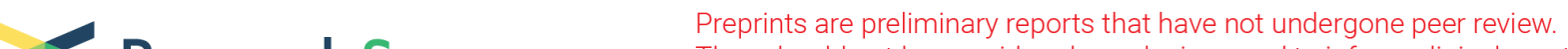 Research Square
They stould not be o onsidideded conclcusivive, sused to inform clinical practice,
or referenced by the media su validated information.
}

\section{Mental health of people living with HIV during the COVID-19 epidemic in Tokyo}

\section{Takahiro Tanaka}

University of Tokyo - Shirogane Campus: Tokyo Daigaku - Shiroganedai Campus

Michiko Koga ( $\nabla$ michiko@ims.u-tokyo.ac.jp )

University of Tokyo - Shirogane Campus: Tokyo Daigaku - Shiroganedai Campus https://orcid.org/0000-0002-3122-9735

\section{Tomoe Senkoji}

University of Tokyo - Shirogane Campus: Tokyo Daigaku - Shiroganedai Campus

\section{Megumi Kubota}

University of Tokyo - Shirogane Campus: Tokyo Daigaku - Shiroganedai Campus

Kazuhiko Ikeuchi

University of Tokyo - Shirogane Campus: Tokyo Daigaku - Shiroganedai Campus

Tadashi Kikuchi

University of Tokyo - Shirogane Campus: Tokyo Daigaku - Shiroganedai Campus

\section{Makoto Saito}

University of Tokyo - Shirogane Campus: Tokyo Daigaku - Shiroganedai Campus

Eisuke Adachi

University of Tokyo - Shirogane Campus: Tokyo Daigaku - Shiroganedai Campus

\section{Takeya Tsutsumi}

University of Tokyo - Shirogane Campus: Tokyo Daigaku - Shiroganedai Campus

\section{Takashi Hosaka}

University of Tokyo - Shirogane Campus: Tokyo Daigaku - Shiroganedai Campus

Hiroshi Yotsuyanagi

University of Tokyo - Shirogane Campus: Tokyo Daigaku - Shiroganedai Campus

\section{Research Article}

Keywords: COVID-19, loneliness, mental health, people living with HIV, Stringency Index

Posted Date: January 10th, 2022

DOI: https://doi.org/10.21203/rs.3.rs-1218745/v1

License: (9) (1) This work is licensed under a Creative Commons Attribution 4.0 International License. Read Full License 
Page $2 / 20$ 


\section{Abstract \\ Background}

The COVID-19 pandemic significantly impacts physical health and mental health. People living with HIV (PLWH) have a higher prevalence of psychiatric disorders than the general population. We examined the mental health of PLWH during the COVID-19 pandemic in Japan.

\section{Methods}

We retrospectively examined the medical records of PLWHs who underwent the Kessler Psychological Distress Scale-10, the Patient Health Questionnaire-9, and the Generalized Anxiety Disorder-7 at an HIV referral hospital in Tokyo. Stringency Index (SI) values were used to measure the intensity of social infection control measures.

\section{Results}

Between February 2020-May 2021, 30 PLWH were examined at least once. The median age was 49 years, 29 PLWH were on antiretroviral therapy, and the median CD4 count was $580.5 / \mu \mathrm{L}$. A total of $30 \%$ of the patients experienced distress, $45 \%$ depression, and $21.4 \%$ generalized anxiety disorder. Fourteen PLWH were taken for a median of four tests (IQR:2.0-7.3) and classified into three types: 1) Moderate distress or mild depression or anxiety at the beginning that decreased gradually (six patients, 43\%), 2) persistent severe distress, but moderate depression or anxiety gradually decreased (six patients, $43 \%$ ), and 3 ) extremely severe distress and depression or anxiety continued to syncope with the SI values (two patients, $14 \%$ ). Having no partners, unemployment, and a history of psychiatric diseases were more commonly observed in the highly severe mental health groups.

\section{Conclusions}

PLWH are more vulnerable to the COVID-19 pandemic than the general population, especially those with substantial social isolation. Attention should be paid to the mental health of PLWH.

\section{Background}

During the COVID-19 pandemic, mental health problems have increased in the general population [1-4]. In the United States (US), 27.7\% of adults showed mental distress in 2020, which was eight times higher than that in 2018 [5]. A meta-analysis reported that COVID-19 caused anxiety, stress, and depression in about $30 \%$ of the general population in Asia and Europe [6]. In Japan, the prevalence of people with depression increased to $18.3 \%$, with at least one of the most common anxiety disorders increasing to $10.6 \%$ during the pandemic [7]. It has been suggested that substantial lockdowns may cause 
psychological distress, depression, and anxiety[8-11], although stringent responses such as social distance and restrictions on going out are necessary to prevent the spread of COVID-19 infection. Furthermore, heavy drinking habits before the pandemic were associated with increased drinking during the COVID-19 pandemic [12]. It has been shown that the COVID-19 pandemic promotes addiction, including smoking, opioid, injection drug use, and alcohol [13]. In France, the Netherlands, and the United Kingdom, the severity of depression and anxiety showed a considerable correlation with Stringency Index (SI) values [14], which indicates the strength of social infection control measures.

Since the early period of this pandemic, studies report that some people are more vulnerable to the psychosocial effects of the pandemic, including people with certain physical diseases, older adults, people at high risk for COVID-19 (including patients with decreased immune function and people living or receiving care in congregate settings), and patients with pre-existing psychiatric disorders such as depression, anxiety disorders, and substance use problems [15].

People living with HIV (PLWH) are known to have a higher prevalence of psychiatric disorders than the general population, even before the COVID-19 pandemic. An extensive survey in the US reported that $29.9 \%$ of male PLWH had mood disorders, including depression, and $33.4 \%$ had anxiety disorders [16]. Moreover, in Japan, $25.7 \%$ of PLWH reported depression, 29.3\% reported anxiety disorders, and the prevalence of mental illness, including substance abuse disorders and adjustment disorders, is 8.6\%-29.6\% [17-19]. In addition, it has been reported that mental health problems among PLWH are associated with decreased quality of life (QOL) [20] and medication adherence [21]. Mental health problems are considered to have a significant impact on the lives and treatment of PLWH. The mental health of this vulnerable group under social restrictions during the COVID-19 pandemic is thus a critical issue.

Therefore, this study retrospectively surveyed the routinely collected medical records of PLWH attending an HIV referral hospital in Tokyo to examine their mental health during the COVID-19 pandemic. Furthermore, we also examined how it changed in response to social infection control policies in Japan and the problems that arose under long-term stress during the COVID-19 pandemic.

\section{Methods}

\section{Study populations}

Social restrictions may affect psychological states due to the COVID-19 pandemic. Therefore, we examined the mental states of PLWH over time by referring to the social restrictions defined by the SI. This retrospective and observational study were performed using routinely collected medical records from February 2020-May 2021 during the COVID-19 surges at an HIV referral hospital in Tokyo, Japan. The subjects were male PLWH receiving regular care, including a mental health care check at the Research Hospital of the Institute of Medical Science at the University of Tokyo.

\section{Measures}


During these COVID-19 surges, three questionnaires were completed to assess mental health: the Kessler Psychological Distress Scale-10 (K10), the Patient Health Questionnaire-9 (PHQ-9), and the Generalized Anxiety Disorder -7 (GAD-7).

\section{K10}

The $\mathrm{K} 10$ is a simple measure of psychological distress, including ten questions about emotional states with five-level responses [22]. It is an assessment of psychological distress over the past 30 days, with a maximum score of 50 indicating highly severe psychological distress and a minimum score of 10 indicating no psychological distress. A total score of 25 or more indicates a severe level of psychological distress, indicating possible mental disorders. A total score of 10-15 points was defined as the mild level of psychological distress, 16-21 points as moderate, 22-29 points as severe, and 30 points or higher as highly severe distress.

\section{PHQ-9}

The PHQ-9 is a 9-item questionnaire that measures the degree of depression severity [23]. Adapted from the Diagnostic and Statistical Manual of Mental Disorders, 4th edition (DSM-IV), the PHQ-9 is comprised of the same diagnostic symptom criteria. Each item is evaluated ranging from 0-3 grade, as the respondent is asked to rate how often each symptom occurred over the last two weeks (0-not at all, 1several days, 2-more than half of the days, and 3-nearly every day), with the total score ranging from 0-27. A total score of 10 or higher on the nine items was defined as moderate or severe depression. The severity of depression was classified 5-9 points as mild (depressive states, subthreshold depression), 10-14 points as moderate, and 15 points or higher as severe.

\section{GAD-7}

The GAD-7 is a 7-item questionnaire to clarify anxiety severity [24]. Each item is evaluated on a severity scale ranging from $0-3$, wherein the respondent is asked to rate how often each symptom occurred over the last two weeks (0-not at all, 1-several days, 2-more than half of the days, and 3-nearly every day), yielding a total score ranging from 0-21. A total score of 10 or higher on the seven items was defined as a generalized anxiety disorder. The severity of anxiety was classified 5-9 points as mild, 10-14 points as moderate, and 15 points or higher as severe.

\section{Assessment of social infection control}

In Tokyo, the first surge of the COVID-19 pandemic was observed from February-June 2020, the second from July-October 2020, the third from November 2020-March 2021, and the fourth from April-May 2021 (Figure 1). The SI of the Oxford COVID-19 Government Response Tracker was used to measure the government's policy response to COVID-19 in Japan. The index records the rigidity of 'Lockdown Style' policies that primarily restrict people's behaviors. It is calculated using all ordinal containment and 
closure policy indicators and recording public information campaigns $[25,26]$. The trend of SI in Japan is shown in Figure 1.

\section{Statistical analysis}

The subjects' demographic, clinical, and clinical psychology test data were summarized using proportions, medians, and interquartile range (IQR). Due to the small sample size, only descriptive statistics were presented.

\section{Results}

\section{Clinical characteristics}

The baseline characteristics of the $30 \mathrm{PLWH}$ are shown in Table 1. All PLWH were male. All but one PLWH were men who have sex with men (MSM). The median age was 49 years (IQR: 42.3-55.0), 29 of 30 patients were on antiretroviral therapy (96.7\%), and 24 were using the integrase-inhibitor-based regimen (80.0\%). The median CD4 count was 580.5/ $\mu$ l (355.0-770.8), and 28 patients had suppressed HIV-RNA quantification (93.3\%). The median time since diagnosis was 10.0 years (8.8-16.0). Eighteen patients were employed (60.0\%), 20 had a partner $(66.7 \%), 12$ were living alone $(40.0 \%)$, and 13 had a history of psychiatric hospital visits (43.3\%).

\section{Mental Health}

We carried K10, PHQ-9, and GAD-7 clinical psychology tests for 30 PLWH during the study period. Nine of $30 \mathrm{PLWH}(30.0 \%)$ exceeded the cut-off score in $\mathrm{K} 10$ and were evaluated to be at the same level of distress as psychiatric disorders. Similarly, 13 of $29 \mathrm{PLWH}(44.8 \%)$, excluding those who did not take the test, exceeded the cut-off score on the PHQ-9 and were evaluated to have depression. Six of 28 PLWH (21.4\%), excluding the two who did not take the test, exceeded the cut-off score on the GAD-7 and were evaluated to have a generalized anxiety disorder. These are shown in Figure 2 (A, B, and $C)$.

\section{The relation between the SI and the mental health of PLWH}

Of the $30 \mathrm{PLWH}, 14 \mathrm{PLWH}$ took the clinical psychology tests several times during the study period (median four times; 2.0-7.3). Fourteen PLWH wished to continue taking the clinical psychology tests. Their median age was 49.5 years (41.5-55.0), as shown in Table1.

The trends in the K10, PHQ-9, and GAD-7 results of the 14 PLWH can be classified into the following three patterns. PLWH whose scores were cut off or more minor in all psychological tests were classified as pattern 1, PLWH whose scores were cut off or more in one of the psychological tests but improved to cut 
off or less were classified as pattern 2, and PLWH who consistently scored cut off or more in all psychological tests were designated as pattern 3.

\section{Pattern 1: The moderate distress or mild depression or anxiety from the first surge decreased gradually (six PLWH042.9\%)}

The median scores of K10 were 19.0, 16.0, 18.0, and 14.5 from the first to the fourth surge, the median scores of PHQ-9 were 7.0, 4.0, 5.0, 2.5, and the median scores of GAD-7 were 6.0, 6.0, 3.0, 3.0, as shown in Figure $3 \mathrm{~A}$. The score of $\mathrm{K} 10$, which was moderate in the first surge, decreased when the SI values decreased in the second surge and increased in the third surge when the SI values increased again. In the fourth surge, where the SI values decreased and then increased, the K10 score decreased significantly and was mild. The PHQ-9 and GAD-7, mild in the first surge, decreased in the second surge when the SI values decreased and remained at the same level in the third surge when the SI values increased. They decreased further during the fourth surge and showed normal. Four patients had a partner, three of whom had no psychiatric history and were employed, and one of whom was employed but had a history of psychiatric hospital visits. Two partnerless patients had no history of psychiatric hospital visits and were either employed or unemployed (Figure 3B). In Pattern 1, only one out of six PLWH did not have a partner and was unemployed, and few patients were lonely or socially isolated.

\section{Pattern 2: The severe distress had not changed, but the moderate depression or anxiety gradually decreased (six PLWH: 42.9\%)}

The median scores of $\mathrm{K} 10$ were 22.0, 18.5, 19.0, and 18.0 from the first to the fourth surge; similarly, the median scores of the PHQ-9 were 11.0, 7.5, 7.0, and 3.0, and the median scores of the GAD-7 were 6.0, 6.0, 3.0, and 3.0, as shown in Figure 3C. The score of $\mathrm{K} 10$, which was severe in the first surge, decreased when the SI values decreased in the second surge and remained almost the same in the third surge when the SI values increased again. Even in the fourth surge, when the SI values decreased and then increased, the score of K10 remained unchanged, showing an intermediate state. The PHQ-9 score, which was moderate in the first surge, decreased in the second surge when the SI values decreased and remained almost the same in the third surge when the SI values increased. In the fourth surge, with periods of high and low SI values, the PHQ-9 score decreased further and showed normal. The GAD-7 score, which showed a mild level in the first surge, did not change in the second surge when the SI values decreased but decreased in the third surge when the SI values increased. It showed normal in the fourth surge. All six of the PLWH had a partner, three of whom had a history of psychiatric hospital visits and were unemployed, two had no history of psychiatric hospital visits and were employed, and one had a history of psychiatric hospital visits and was employed (Figure 3D). In Pattern 2, all patients had a partner, and none of them were considered lonely or socially isolated.

\section{Pattern 3: The highly severe distress and severe depression or anxiety had shifted correspondingly up and down in the SI values (two PLWH: 14.3\%)}


The median scores of K10 were 32.0, 39.0, 42.0, and 33.0 from the first to the fourth surge, the median scores of PHQ-9 were 18.5, 16.0, 21.0, and 17.0, and the median scores of GAD-7 were 15.5, 13.0, 17.0, and 13.0, as shown in Figure 3C. The score of K10, the most severe in the first surge, increased when the SI values decreased in the second surge and further increased in the third surge when the SI values were the highest. In the fourth surge, where the SI values decreased and then increased, the test score decreased but remained the most severe condition. The PHQ-9 and GAD-7 scores showed that the severity of the first surge decreased in the second surge when the SI value decreased and increased again in the third surge when the SI values increased. The score decreased in the fourth surge when the SI values were high and low, respectively. In the fourth surge, the PHQ-9 showed severity, GAD-7 showed moderate, and PHQ-9 and GAD-7 shifted up and down, respectively, in the SI values (Figure 3E). These two PLWH were unemployed and had a history of psychiatric hospital visits, and one of them had a partner but was the poorest in all clinical psychology tests from the beginning of the pandemic (Figure 3F). In Pattern 3, they all had characteristics that made them prone to loneliness or social isolation.

\section{Discussion}

We found that during the COVID-19 pandemic, $30 \%$ of PLWH were highly stressed, $44.8 \%$ had depression, and $21.4 \%$ had anxiety disorders. Additionally, we found that the depression and anxiety disorders of some PLWH moved up and down correspondingly to the social infection control measures during the COVID-19 pandemic. This is the first study to evaluate the change in the mental health of PLWH while the COVID-19 pandemic prolongs and the strength of social infection control changes over time. The PLWH feel isolated because they do not have a partner, live alone, do not work in society, or have a history of mental illness, and are expected to experience intense distress, severe depression, and anxiety disorders due to isolation from society.

Despite the small number of subjects in this study, the median age, the treatment rate of ART, the effectiveness of suppressed HIV-RNA quantification, and the rate of using an integrase inhibitor regimen, working, and living alone are not very different from the average PLWH in Tokyo [27]. These patients also have a well-suppressed HIV viral load and high CD 4 counts with long ART, and the medical condition seems to be well-being.

In another Japanese internet-based survey using the Kessler 6 (K6) in the general population of Japan, a test like the K10, severe psychological distress was reported in $9.3-11.3 \%$ of the population during the COVID-19 pandemic [28]. In this study, 30\% of PLWH were stressed, which was much higher than in the general Japanese population because PLWH are particularly vulnerable to stress, tend to be isolated from society due to prejudice and have a high prevalence of mental illness. Of the 30 patients included in this study, $43.3 \%$ had a history of psychiatric visits. It was suggested that during the COVID-19 pandemic, PLWH might present more substantial mental distress than the general population.

During the COVID-19 pandemic, a multinational online survey of PLWH reported depression in $7.1 \%-41.0 \%$ and generalized anxiety disorder in $14.3 \%-39.8 \%$ [29]. Since our study reported depression in $44.8 \%$ and 
generalized anxiety disorder in $21.4 \%$, the prevalence of depression and generalized anxiety disorder in PLWH was approximately the same in other countries. In the general population of Japan, depression and generalized anxiety disorder were reported to be $18.3 \%$ and $10.6 \%$, respectively, during the COVID-19 pandemic [7]. PLWH living in Japan may be far more stressed and more than twice as depressed and anxious as people without HIV infection. Moreover, an online survey of $98 \mathrm{PLWH}$ in Italy during the COVID-19 pandemic reported that $45 \%$ showed psychological distress measured by the Impact of Event Scale-Revised (ISE-R), which was higher than that in our study [30]. A simple comparison cannot be made because the psychological tests were different. This result may be because, in Italy, the number of COVID19 patients from the early stages of the pandemic was much larger than that in Japan, and more rigorous social infection control measures were in place than in Japan[25, 31]. Our study implied that the psychological distress of PLWH might increase as social infection control becomes more rigid, and we can infer that this is not limited to Japan.

Regarding mental health trends, 85.8\% of PLWH (Pattern 1: $42.9 \%$ and Pattern 2: $42.9 \%$ ) improved regardless of the change in social infection control. The results are not similar to previous studies that showed a correlation between SI values and mental health trends [29]. However, $14.2 \%$ of PLWH had worse mental health, similar to previous studies. Therefore, it was speculated that the social stringency of COVID-19 may be related to the mental health of certain PLWH.

Notably, PLWH with a partner showed improvement from severe distress, moderate depression, and anxiety in the present study. In the general population, loneliness aggravates mental health and has been linked to increased depression, generalized anxiety disorder, and suicidal ideation [32]. Employment and living with a partner may be a protective factor for mental health problems in the COVID-19 pandemic [33]. It is suggested that intimate connections with others may act as a protective factor for mental health problems among PLWH. In addition, it is important that PLWH without improvement had a psychiatric history, were unemployed, and were in a group that suggested loneliness or social isolation. A study conducted by the University of Chicago's National Opinion Research Center noted that depression and acute stress reactions after the COVID-19 pandemic were associated with psychiatric history before the pandemic [34]. In an environment including loneliness, home isolation, and social distance, patients with a psychiatric history are more susceptible to mental health deterioration [35]. The PLWH initially vulnerable to stress may develop mental health problems if combined with other several factors more susceptible to vulnerability. In Japan, a mental health study conducted during the second surge of the COVID-19 outbreak reported that unemployment exacerbated depression [36]. This suggests that Ioneliness or social isolation may aggravate mental health even during the COVID-19 pandemic in Japan. Furthermore, due to internalized stigma, PLWH in Japan may fear adverse reactions from others and avoid interaction with others [37]. The combination of unemployed status and social infection control measures, which may lead to more time spent alone, may have accentuated loneliness or social isolation in patients with a psychiatric history. PLWH, who are more likely to be unemployed or have a history of psychiatric hospital visits, may need to pay attention to their mental health during the COVID-19 pandemic in Japan. Concern about the recurrence of mental illness and restriction of the movement 
included in social infection control measures may also lead to interruptions in medication and counseling [15]; therefore, early intervention may be necessary for them[38-40].

This study has several limitations that require careful interpretation of the results. The sample size of this study was insufficient and may not necessarily represent PLWH in Japan. In addition, since the data were collected from a single institution, there are limitations in generalizing this data. Thirty and 14 PLWH who underwent continuous psychological tests were randomly selected; however, statistical analysis could not be performed due to the small number of subjects. Future studies must increase the number of subjects and clarify the factors that affect mental health and its transition. Furthermore, this study measured mental health problems using a self-administered questionnaire. Since psychological questionnaire tests can deliberately distort answers, the testing method may cause a bias. Moreover, we limited the mental health problems to distress, depression, and generalized anxiety disorder and did not investigate obsessive-compulsive disorder or post-traumatic stress disorder (PTSD). In addition, this study did not consider income or unemployment during the spread of the COVID-19 pandemic, which might have influenced mental health problems. Finally, the course of addiction, such as smoking, opioids, injection drug use, and alcohol, is unknown.

Further quantitative and qualitative studies on mental health symptoms, their trends, and influencing factors in this study can help examine effective intervention methods during the spread of COVID-19. The findings of this study might contribute to the mental support at large and to society.

Under COVID-19 infection, PLWH in Tokyo are under severe stress. Early intervention in mental health is essential, especially for PLWH who feel lonely and socially isolated without a partner or are unemployed. An unexpected outbreak of infectious diseases must occur again. An essential insight we have gained from the COVID-19 pandemic is that early mental intervention of PLWH is necessary for this kind of crisis.

\section{Conclusions}

We showed that under the COVID-19 epidemic, $30 \%$ of PLWH are highly stressed, $44.8 \%$ have depression, and $21.4 \%$ have anxiety disorders. Additionally, If PLWH is apt to feel isolated because they do not have a partner, live alone, or do not work in society, or if they have a history of mental illness, PLWH is vulnerable to social infection control.

\section{Declarations}

\section{Availability of data and materials}

The datasets generated and analyzed during the current study are available from the corresponding author on reasonable request.

\section{Acknowledgements}


Tomoko Sato and Akari Fukuda assisted with this study. Overall, these patients contributed to this study. All data were generated by individuals who spent time in the hospital. We would like to thank Editage (www.editage.com) for English language editing.

\section{Funding}

This study was supported in part by research grants from a Health Labor Sciences research grant (grant number R3-AIDS- general-21HB2005).

Author information

Affiliations

Department of Infectious Diseases and Applied Immunology, Hospital of the Institute of Medical Science, University of Tokyo, 4-6-1, Shirokanedai, Minato-ku, Tokyo 108-8639, Japan

Takahiro Tanaka, Michiko Koga, Kazuhiko Ikeuchi, Tadashi Kikuchi, Eisuke Adachi, Takashi Hosaka \& Hiroshi Yotsuyanagi

Department of Psychology, St-Pierre Hospital, 786-7, Kamisano-cho, Takasaki-shi, Gunma 370-0857, Japan

Takahiro Tanaka

Division of Infectious Diseases, Advanced Clinical Research Center, Institute of Medical Science, University of Tokyo, 4-6-1, Shirokanedai, Minato-ku, Tokyo 108-8639, Japan

Michiko Koga, Tomoe Senkoji, Megumi Kubota, Makoto Saito, Takeya Tsutsumi \& Hiroshi Yotsuyanagi

Department of Psycho-Oncology, HOSAKA Psycho-Oncology Clinic, \#A 6F,

Tsukiji Asahikawa Bldg. 11-3 Akashi-cho,Chuo-ku, Tokyo, 104-0044 Japan

Takashi Hosaka

Authors' contributions

T. T., M. K., and T. H. conceived the concept of this study, were responsible for the study design and collected the data. All authors interpreted the collected data, drafted the manuscript, and provided feedback. All authors critically read the drafted manuscript and approved the final version. 


\section{Ethics Declarations}

\section{Ethics approval and consent to participate}

This study was a retrospective analysis of clinical records and was approved by the Ethics Review Committee at the University of Tokyo (approval No.: 30-110-B20190404) and conformed to the provisions of the Declaration of Helsinki. The data were de-identified and anonymous.

\section{Consent for publication}

All author have given consent for publication.

\section{Competing interests}

The authors declare that they have no competing interests.

\section{Abbreviations}

AIDS, acquired immunodeficiency syndrome; HIV, human immunodeficiency virus; PLWH, People living with HIV; IMSUT, Institute of Medical Science, University of Tokyo; IQR, interquartile range; MSM, men who have sex with men; K10, the Kessler Psychological Distress Scale-10; PHQ-9, the Patient Health Questionnaire-9; GAD-7, the Generalized Anxiety Disorder-7; DSM-IV, Diagnostic and Statistical Manual of Mental Disorders 4th edition; SI, the Stringency Index; PTSD, Post-traumatic Stress Disorder

\section{References}

[1] Mo, G.H., Wang, Z.X., Chen, X.S. et al. The prognosis and prevention measures for mental health in COVID-19 patients: through the experience of SARS. BioPsychoSocial Med 14, 22 (2020). https://doi.org/10.1186/s13030-020-00196-6

[2] Betty P, Carol SN. Mental Health and the Covid-19 Pandemic. N Engl J Med 2020;383(6):510512.https://doi.org/10.1056/NEJMp2008017

[3] Moreno C, Wykes T, Galderisi S, et al. How mental health care should change as a consequence of the COVID-19 pandemic [published correction appears in Lancet Psychiatry. 2021 Jul;8(7):e16]. Lancet Psychiatry 2020;7(9):813-824. https://doi.org/10.1016/S2215-0366(20)30307-2

[4] Galea S, Merchant RM, Lurie N. The Mental Health Consequences of COVID-19 and Physical Distancing: The Need for Prevention and Early Intervention. JAMA Intern Med 2020;180(6):817-818. https://doi.org/10.1001/jamainternmed.2020.1562 
[5] Twenge JM, Joiner TE. Mental distress among U.S. adults during the COVID-19 pandemic. J Clin Psychol 2020;76(12):2170-2182. https://doi.org/10.1002/jclp.23064

[6] Salari N, Hosseinian-Far A, Jalali R, et al. Prevalence of stress, anxiety, depression among the general population during the COVID-19 pandemic: a systematic review and meta-analysis. Global Health 2020;16(1):57. https://doi.org/10.1186/s12992-020-00589-w

[7] Ueda M, Nordström R, Matsubayashi T. Suicide and mental health during the COVID-19 pandemic in Japan. J Public Health (Oxf) 2021;fdab113. https://doi.org/10.1093/pubmed/fdab113

[8] Voss G, Paiva AF, Delerue Matos A. A Study of the Association between the Stringency of Covid-19 Government Measures and Depression in Older Adults across Europe and Israel. Int J Environ Res Public Health 2021;18(15):8017. https://doi.org/10.3390/ijerph18158017

[9] Lee JH, Lee H, Kim JE, Moon SJ, Nam EW. Analysis of personal and national factors that influence depression in individuals during the COVID-19 pandemic: a web-based cross-sectional survey. Global Health. 2021;17(1):3. https://doi.org/10.1186/s12992-020-00650-8

[10] Tull MT, Edmonds KA, Scamaldo KM, Richmond JR, Rose JP, Gratz KL. Psychological Outcomes Associated with Stay-at-Home Orders and the Perceived Impact of COVID-19 on Daily Life. Psychiatry Res 2020;289:113098. https://doi.org/10.1016/j.psychres.2020.113098

[11] Benke C, Autenrieth LK, Asselmann E, Pané-Farré CA. Lockdown, quarantine measures, and social distancing: Associations with depression, anxiety and distress at the beginning of the COVID-19 pandemic among adults from Germany. Psychiatry Res 2020;293:113462.

https://doi.org/10.1016/j.psychres.2020.113462

[12] Neill E, Meyer D, Toh WL, et al. Alcohol use in Australia during the early days of the COVID-19 pandemic: Initial results from the COLLATE project. Psychiatry Clin Neurosci 2020;74(10):542-549. https://doi.org/10.1111/pcn.13099

[13] Mahua JD, Ritwik G, Subham C, Payel B, Subhankar C, Souvik D. COVID-19 and addiction. Diabetes Metab Syndr 2020;14(5):817-823. https://doi.org/10.1016/j.dsx.2020.06.008

[14] Organization for Economic Co-operation and Development (OECD): TRACKING CORONAVIRUS (COVID-19) CONTRIBUTING TO A GLOBAL EFFORT, Tackling the mental health impact of the COVID-19 crisis: An integrated, whole-of-society response. [Accessed 27 August 2021] Available from https://read.oecd-ilibrary.org/view/?ref=1094_1094455-bukuf1f0cm\&title=Tackling-the-mental-healthimpact-of-the-COVID-19-crisis-An-integrated-whole-of-societyresponse\&_ga=2.41681378.1581547161.1630208047-696326152.1572945659

[15] Dubey S, Biswas P, Ghosh R, et al. Psychosocial impact of COVID-19. Diabetes Metab Syndr 2020;14(5):779-788. https://doi.org/10.1016/j.dsx.2020.05.035 
[16] Lopes M, Olfson M, Rabkin J, et al. Gender, HIV status, and psychiatric disorders: results from the National Epidemiologic Survey on Alcohol and Related Conditions. J Clin Psychiatry 2012;73(3):384-391. https://doi.org/10.4088/JCP.10m06304

[17] HIV Futures Japan Project: 2nd Web-based survey for HIV-positive people. [Accessed 17 July 2021] Available from https://survey.futures-japan.jp/doc/summary_2nd_all.pdf (in Japanese)

[18] Watanabe A, Nishijima T, Takahashi T et al. The Prevalence and Characteristics of Psychiatric Disorders among HIV-Infected Patients in the ART Era. The journal of AIDS research 2018; 20:47-52. (in Japanese)

[19] Hukunishi I, Hirabayashi N, Matsumoto T, Yamanaka K, Hosaka T, Horikawa N. Psychiatric disorders in HIV-positive patients; a study of the prevalence rate of psychiatric disorders and its relationship with an immunological indicator. Japanese journal of clinical psychiatry 1999;28(10):1233-1242. (in Japanese)

[20] Sherbourne CD, Hays RD, Fleishman JA, et al. Impact of psychiatric conditions on health-related quality of life in persons with HIV infection. Am J Psychiatry 2000;157(2):248-254.

https://doi.org/10.1176/appi.ajp.157.2.248

[21] Whetten K, Reif S, Whetten R, Murphy-McMillan LK. Trauma, mental health, distrust, and stigma among HIV-positive persons: implications for effective care. Psychosom Med 2008;70(5):531-538. https://doi.org/10.1097/PSY.0b013e31817749dc

[22] Kessler RC, Barker PR, Colpe LJ, et al. Screening for serious mental illness in the general population. Arch Gen Psychiatry 2003;60(2):184-189. https://doi.org/10.1001/archpsyc.60.2.184

[23] Kroenke K, Spitzer RL, Williams JB. The PHQ-9: validity of a brief depression severity measure. J Gen Intern Med 2001;16(9):606-613. https://doi.org/10.1046/j.1525-1497.2001.016009606.x

[24] Spitzer RL, Kroenke K, Williams JB, Löwe B. A brief measure for assessing generalized anxiety disorder: the GAD-7. Arch Intern Med 2006;166(10):1092-1097.

https://doi.org/10.1001/archinte.166.10.1092

[25] Oxford COVID-19 Government Response Tracker, Blavatnik School of Government, University of Oxford. [Accessed 17 July 2021] Available from https://www.bsg.ox.ac.uk/research/researchprojects/covid-19-government-response-tracker

[26] Hale T, Angrist N, Goldszmidt R, et al. A global panel database of pandemic policies (Oxford COVID-19 Government Response Tracker). Nat Hum Behav 2021;5(4):529-538.

https://doi.org/10.1038/s41562-021-01079-8

[27] Komatsu K, Kimura S, Kiryu Y, et al. Detailed analysis of social support and proactive coping with depressive symptoms in Japanese HIV-infected individuals. AIDS Care. 2021;1-9.

https://doi.org/10.1080/09540121.2021.1934382

Page 14/20 
[28] Kikuchi H, Machida M, Nakamura I, et al. Changes in Psychological Distress During the COVID-19 Pandemic in Japan: A Longitudinal Study. J Epidemiol 2020;30(11):522-528.

https://doi.org/10.2188/jea.JE20200271

[29] Siewe Fodjo JN, Faria de Moura Villela E, Van Hees S, Vanholder P, Reyntiens P, Colebunders R. Follow-Up Survey of the Impact of COVID-19 on People Living with HIV during the Second Semester of the Pandemic. Int J Environ Res Public Health 2021;18(9):4635. https://doi.org/10.3390/ijerph18094635

[30] Delle Donne V, Ciccarelli N, Massaroni V, et al. Psychological distress during the initial stage of the COVID-19 pandemic in an Italian population living with HIV: an online survey. Infez Med 2021;29(1):5464.

[31] World Health Organization(WHO)囚Coronavirus (COVID-19) Dashboard, Italy. [Accessed 27 August 2021] Available from https://covid19.who.int/region/euro/country/it

[32] Beutel ME, Klein EM, Brähler E, et al. Loneliness in the general population: prevalence, determinants and relations to mental health. BMC Psychiatry. 2017;17(1):97. https://doi.org/10.1186/s12888-017$1262-x$

[33] Li LZ, Wang S. Prevalence and predictors of general psychiatric disorders and loneliness during COVID-19 in the United Kingdom. Psychiatry Res. 2020;291:113267. https://doi.org/10.1016/j.psychres.2020.113267

[34] Holman EA, Thompson RR, Garfin DR, Silver RC. The unfolding COVID-19 pandemic: A probabilitybased, nationally representative study of mental health in the United States. Sci Adv.

2020;6(42):eabd5390. https://doi.org/10.1126/sciadv.abd5390

[35] Rodríguez-Fernández P, González-Santos J, Santamaría-Peláez M, Soto-Cámara R, SánchezGonzález E, González-Bernal JJ. Psychological Effects of Home Confinement and Social Distancing Derived from COVID-19 in the General Population-A Systematic Review. Int J Environ Res Public Health 2021;18(12):6528.

[36] Fukase $Y$, Ichikura $K$, Murase $H$, Tagaya $H$. Depression, risk factors, and coping strategies in the context of social dislocations resulting from the second surge of COVID-19 in Japan. BMC Psychiatry 2021;21(1):33. https://doi.org/10.1186/s12888-021-03047-y

[37] Komatsu K, Kojima K. Treatment of Psychiatric Disorders and Psychological Problems among People Living with HIV/AIDS. The journal of AIDS research 2016;18 (3):183-196. (in Japanese)

[38] Ghio L, Gotelli S, Marcenaro M, Amore M, Natta W. Duration of untreated illness and outcomes in unipolar depression: a systematic review and meta-analysis. J Affect Disord 2014;152-154:45-51. https://doi.org/10.1016/j.jad.2013.10.002 
[39] Marco JH, Alonso S, Andani J. Early intervention with cognitive behavioral therapy reduces sick leave duration in people with adjustment, anxiety and depressive disorders. J Ment Health 2020;29(3):247255.https://doi.org/10.1080/09638237.2018.1521937

[40] Kameno Y, Hanada A, Asai D, et al. Individual psychotherapy using psychological first aid for frontline nurses at high risk of psychological distress during the COVID-19 pandemic. Psychiatry Clin Neurosci 2021;75(1):25-27. https://doi.org/10.1111/pcn.13170

\section{Tables}

Table 1. Baseline characteristics of PLWH who took the clinical psychology tests 
Baseline characteristic

All patients

Patients whose progress was surveyed

$\mathrm{n}=30 \quad \mathrm{n}=14$

$\mathrm{Age}^{\dagger}$

$49.0 \quad(42.3-55.0) \quad 49.5$

$(39.8-55.8)$

History of AIDS-defining illness

5

$(16.7 \%)$

2

$(14.3 \%)$

HIV transmission route

\begin{tabular}{|c|c|c|c|c|}
\hline Men who have sex with men & 29 & $(96.7 \%)$ & 14 & $(100.0 \%)$ \\
\hline Heterosexuals & 1 & $(3.3 \rrbracket)$ & 0 & \\
\hline Current ART use & 29 & $(96.7 \%)$ & 13 & $(92.9 \%)$ \\
\hline \multicolumn{5}{|l|}{ Current antiretroviral treatment } \\
\hline NNRTI-based regimen & 2 & $(6.7 \%)$ & 2 & $(14.3 \%)$ \\
\hline PI-based regimen & 3 & $(10.0 \%)$ & 1 & $(7.1 \%)$ \\
\hline INSTI-based regimen & 24 & $(80.0 \%)$ & 10 & $(71.4 \%)$ \\
\hline HIV viral load $<50$ copies $/ \mathrm{mL}$ & 28 & $(93.3 \%)$ & 13 & $(92.9 \%)$ \\
\hline $\operatorname{CD} 4$ count $(/ \mu \mathrm{l})^{\dagger}$ & 580.5 & $\begin{array}{l}(355.0- \\
770.8)\end{array}$ & 682.0 & $(370.0-780.3)$ \\
\hline $\operatorname{CD} 8$ count $(/ \mu \mathrm{l})^{\dagger}$ & 680.5 & $\begin{array}{l}(560.0- \\
913.3)\end{array}$ & 692.0 & $(627.5-940.0)$ \\
\hline Time since diagnosis (years) $^{\dagger}$ & 10.0 & $(8.8-16.0)$ & 10.5 & $(8.8-14.5)$ \\
\hline Employed & 18 & $(60.0 \%)$ & 8 & $(57.1 \%)$ \\
\hline Married/having a partner & 20 & $(66.7 \%)$ & 11 & $(78.6 \%)$ \\
\hline Living alone & 12 & $(40.0 \%)$ & 5 & $(35.7 \%)$ \\
\hline $\begin{array}{l}\text { History of psychiatric hospital } \\
\text { visits }\end{array}$ & 13 & $(43.3 \%)$ & 7 & $(50.0 \%)$ \\
\hline
\end{tabular}

Educational level

\begin{tabular}{lllll} 
junior high school graduate & 1 & $(3.3 \%)$ & 1 & $(7.1 \%)$ \\
\hline High school graduate & 11 & $(36.7 \%)$ & 3 & $(21.4 \%)$ \\
\hline wo-year college graduate & 4 & $(13.3 \%)$ & 2 & $(14.3 \%)$ \\
\hline Bachelor's degree or higher & 14 & $(46.7 \%)$ & 8 & $(57.1 \%)$
\end{tabular}

People with a diagnosis of substance 
use-related disorders

$\begin{array}{llll}\text { Drug-use-related disorders } & 2 & (6.7 \%) & 0 \\ \text { Alcohol-use-related disorders } & 1 & (3.3 \%) & 0\end{array}$

† Median (interquartile range)

Abbreviations: AIDS, acquired immunodeficiency syndrome; ART, antiretroviral therapy; HIV, human immunodeficiency virus; NNRTI, non-nucleoside reverse transcriptase inhibitor; PI, protease inhibitor; INSTI, integrase strand transfer inhibitor

\section{Figures}

Figure 1.

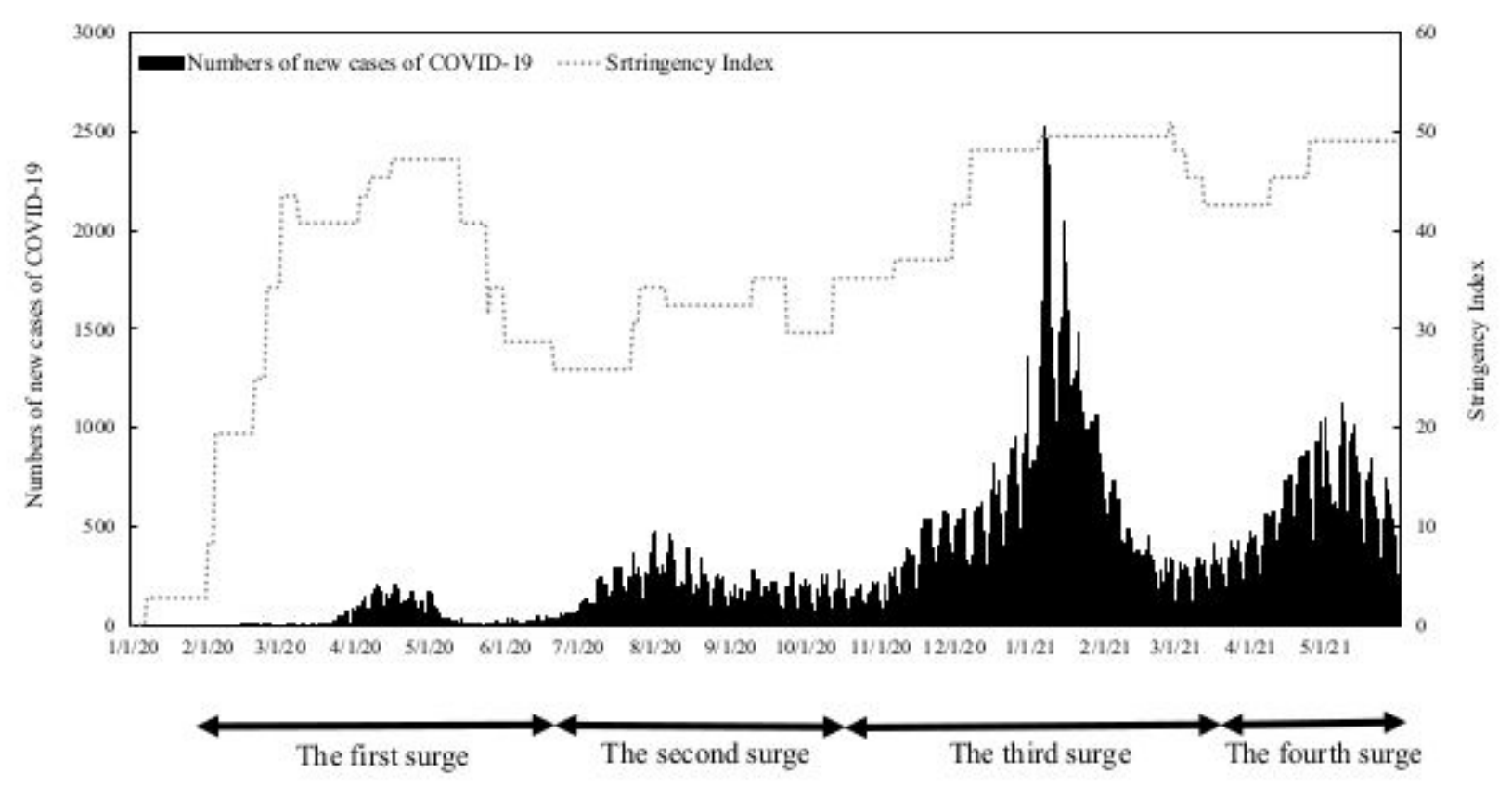

Figure 1

Number of new cases of COVID-19 and timing of the pandemic in Tokyo

Information related to COVID-19 provided by NHK (Japan Broadcasting Corporation) and the Stringency Index derived from the Oxford COVID-19 Government Response Tracker. 
Figure 2.

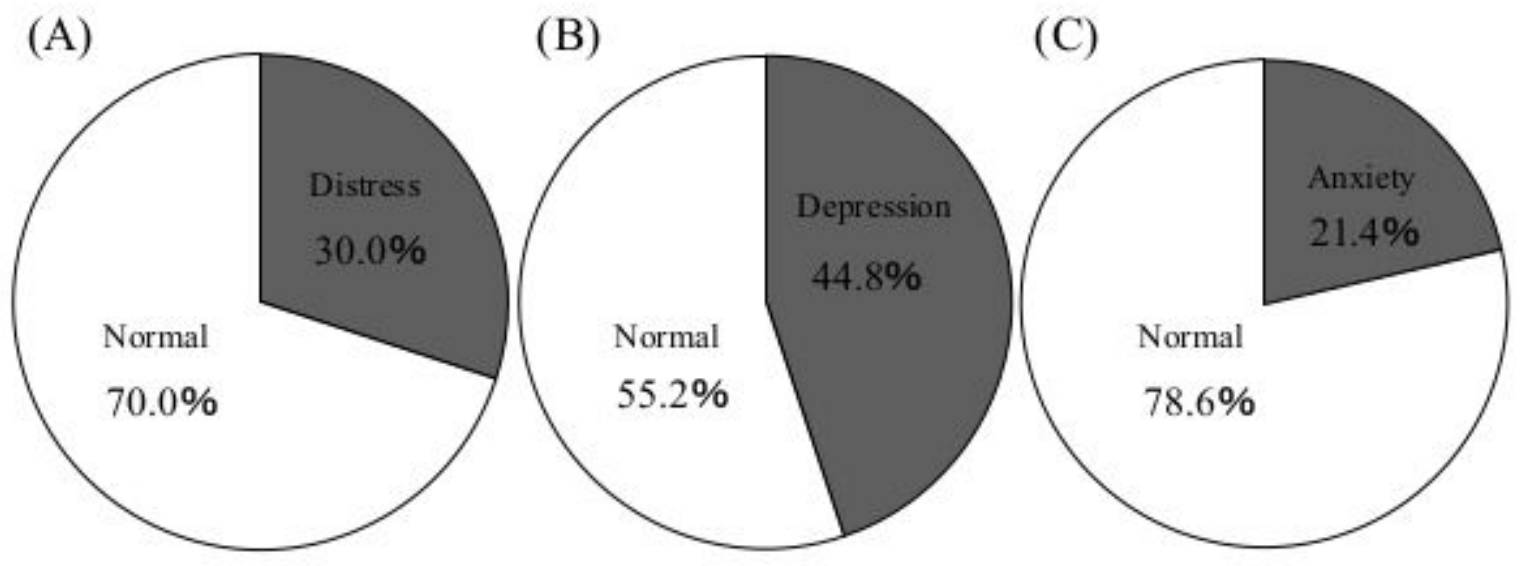

Figure 2

Prevalence of distress, depression, and generalized anxiety disorder assessed at first psychological test $(n=30)$. The distress, depression, and anxiety were shown using K10, PHQ-9, and GAD-7 (A, B, C). 
Figure 3.

(A)

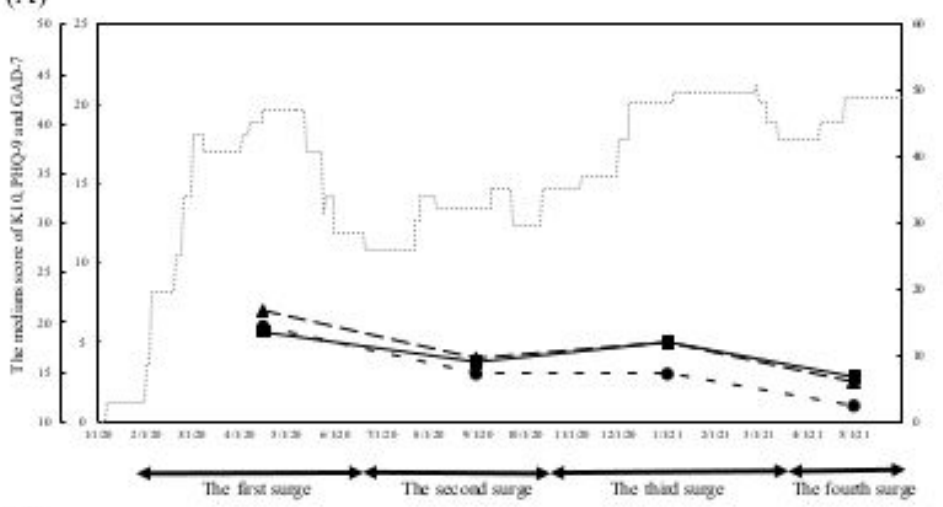

(C)

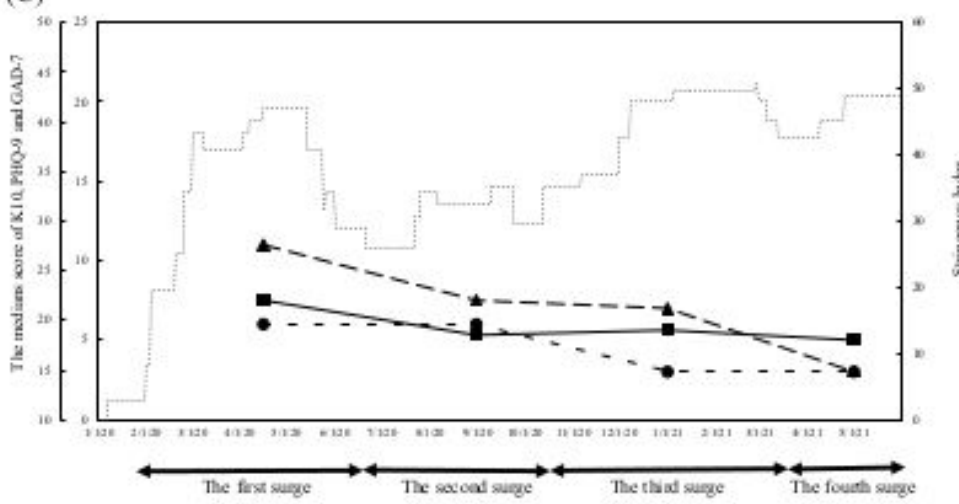

(E)

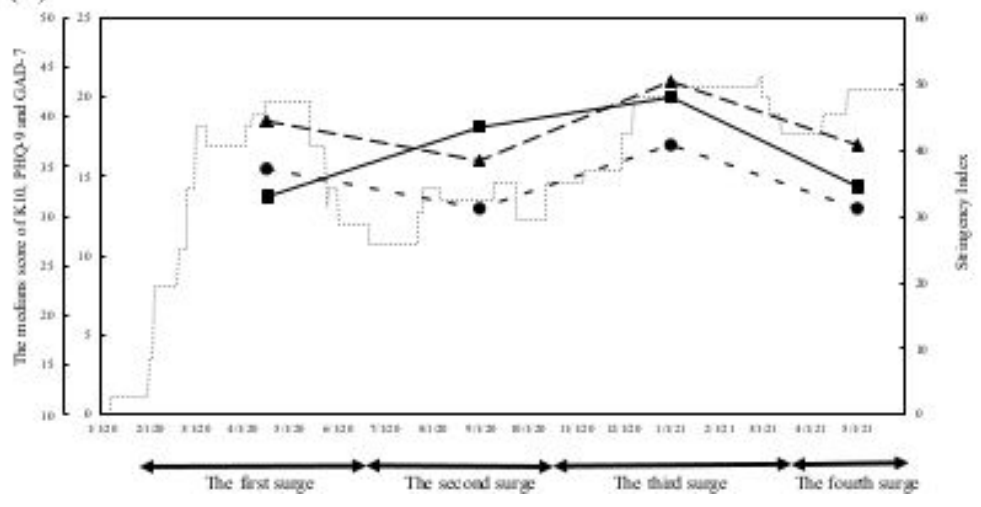

(B)

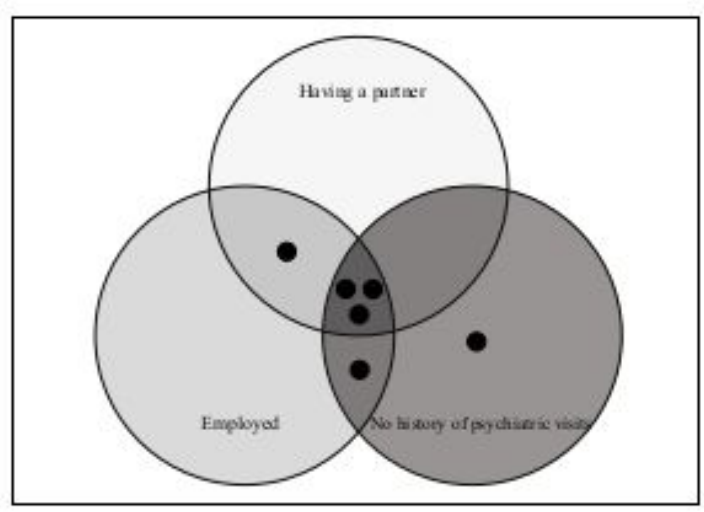

(D)

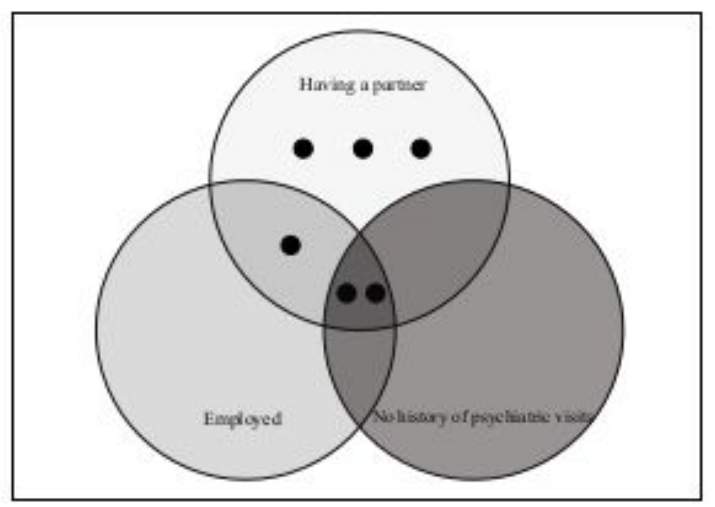

(F)

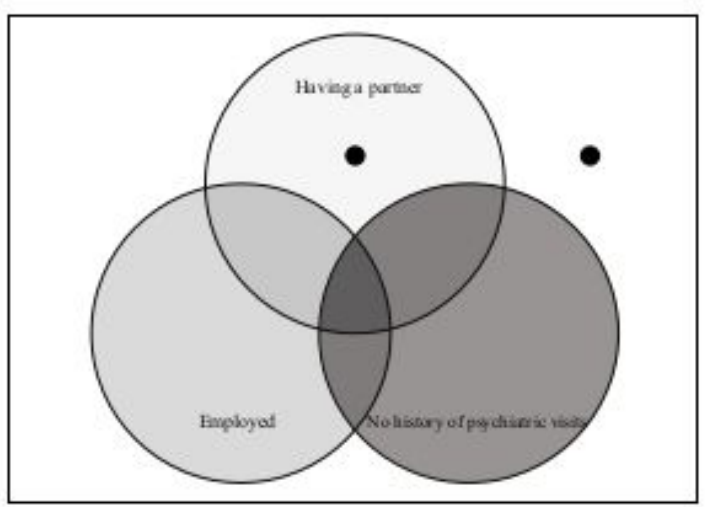

Figure 3

Changes in clinical psychology test values

K10, the Kessler Psychological Distress Scale-10; PHQ-9, the Patient Health Questionnaire-9; GAD-7, the Generalized Anxiety Disorder-7. () Median K10. ( ) The median PHQ-9. () Median GAD-7 score. Stringency Index ( ) (A) The moderate distress or mild depression/anxiety from the first surge (Pattern 1), Overall median K10, PHQ-9 and GAD-7 (interquartile range) = 17.5(15.0-20.0), 5.0(4.0-6.0) and 3.0(1.0-6.0). (C) Severe distress or moderate depression/anxiety during the observation period (pattern 2), overall median K10, PHQ-9, and GAD-7 (interquartile range) $=20.0$ [16.8-23.0), 7.5(4.0-10.8], and 5.5 [3.0-8.0]). (E) The most severe distress and severe depression/anxiety from the first surge (pattern 3), overall median K10, PHQ-9, and GAD-7 (interquartile range) $=39.0(32.5-41.0), 19.0(16.5-20.0)$ and 15.0(13.3-16.8] ). (B) (D) (F) Distribution of PLWH in patterns 1,2 , and 3 , respectively. 